set of subcortical and limbic regions in which opposite changes are found.

Conclusions: The pathophysiology of major depressive disorder involves a complicated series of networks of frontal, temporal-parietal cortical and limbic brain regions and the cerebellum. Questions remain as to whether one or other of these networks play a primary role in the etiology of the disorder.

\section{An fMRI study of the effects of low- and high-frequency transcranial magnetic stimulation treatment in depression}

\author{
P Fitzgerald ${ }^{1,2}$, A Srithiran ${ }^{1,2}, J_{\text {Benitez }}^{1,2}$, J Kulkarni ${ }^{1,2}$, \\ G Egan ${ }^{3}$
}

'Alfred Psychiatry Research Centre, The Alfred; 'Monash University Department of Psychological Medicine; and ${ }^{3}$ Howard Florey Institute, The University of Melbourne, Melbourne, Australia

Objective: The study aimed to explore the effects of high-frequency, left-sided repetitive transcranial magnetic stimulation(rTMS)(HFL-TMS) and low-frequency stimulation to the right prefrontal cortex (LFR-TMS of HFL-TMS) using functional magnetic resonance imaging (fMRI) before and after a course of rTMS in patients with treatment-resistant depression (TRD).

Methods: The study was a randomized parallel before and after trial using fMRI to study the effects of 3 weeks of daily HFL-TMS and LFR-TMS treatment. Twenty-six patients with TRD underwent rTMS treatment and were scanned with fMRI during a planning task pretreatment and after 3 weeks.

Results: There was a significant reduction in depression severity for patients in both treatment groups $[\mathrm{F}(1,24)=17.5, P=0.05)$. Responders to HFLTMS showed an increase in task-related activation in prefrontal regions bilaterally. In contrast, responders to LFR-TMS showed a decrease in bilateral prefrontal activity. There were also differences in pretreatment scans between responders and nonresponders.

Conclusions: Changes in task-related brain activation produced by HFL-TMS and LFR-TMS occur bilaterally in frontal brain regions but are opposite in direction, with high-frequency stimulation increasing and lowfrequency stimulation decreasing task-related activation.

\section{Outcome in a specialist referral clinic for mood disorders: a qualitative and quantitative review}

\author{
K Fletcher ${ }^{1,2}$, G Parker ${ }^{1,2}$, H Brotchie ${ }^{1,2}$, M Hyett $^{1,2}$, \\ M Barrett ${ }^{2}$
}

'School of Psychiatry, University of New South Wales; and ${ }^{2}$ The Black Dog Institute, Sydney, Australia

Background: The Black Dog Institute Depression Clinic provides comprehensive clinical assessment and management strategies, operating to a subtyping diagnostic model. The study aimed to determine whether baseline clinical assessment was predictive of short-term outcome for patients referred with a depressive disorder. Factors contributing to outcome were identified, and the utility of a subtyping approach was discussed.

Methods: A consecutive series of 85 patients referred to the clinic completed the computerized mood assessment program, followed by interview with the assessing psychiatrist. Prognostic judgments were made reflecting clinical factors (eg disorder type, previous therapy response). Quantitative and qualitative analyses of other contributory factors were undertaken to assess impact on outcome.

Results: Global assessment of outcome at baseline was predictive of short-term outcome, while outcome trajectories were influenced for those who did not receive (or were unable to continue with) recommended treatments. Comparably high rates of improvement were evident in those with bipolar, melancholic and nonmelancholic subtypes, and somewhat lower in those diagnosed with 'secondary depression'. Other factors influencing outcome included referral source (ie psychiatrist vs. general practitioner), degree of recommendation uptake and implementation of psychotropic drug strategies.

Conclusions: Improvement rates were high in a clinic weighting a subtyping diagnostic approach to shaping pluralistic management plans. Nonetheless, the absence of a comparator service disallows firm conclusions. These results will guide further definitive study designs.

\section{First-episode psychosis in the community in NSW: detection and service utilization}

\section{P Fogarty, G Sara \\ InforMH, NSW Department of Health, Sydney, Australia}

Background: New South Wales has adopted an early psychosis (EP) program based on research that early intervention improves mental health outcomes. EP 'flags' recorded at community contacts are reportedly underused, making it difficult to analyze EP pathways and outcomes. This study aims to measure the number of first-episode psychosis cases in 2004-2005, examine the performance of the EP flags and investigate differences in service utilization. 